\title{
XVII.
}

Aus der medicinischen Klinik in Strassburg.

\section{Ueber das Zittern bei Paralysis agitans.}

\author{
Von \\ Dr. D. Gerhardt, \\ Privatdocent und Assistent der Klinik.
}

(Nach einem Vortrag, gehalten auf der 20. Wanderversammlung südwestdeutscher Neurologen und Irrenärzte zu Baden-Baden, Juni 1896.)

(Mit 1 Curve.)

'Die Charcot'sche Lehre, dass multiple Sklerose und Schüttellähmung durch die Art des Zitterns gut von einander zu scheiden seien, hat sich rasch verbreitet. Dass bei jener ein ausgesprochener Intentionstremor bestehe, der in der Ruhe ganz aufhöre, dass dagegen die Paralysis agitans gekennzeichnet sei durch fortwährende, auch bei völliger Ruhe anhaltende Oscillationen, welche durch Bewegungen unterdrückt werden können, wurde seither allgemein angenommen, also dort Tremor bei Bewegungen, in der Ruhe abnehmend, hier in der Ruhe, bei Bewegungen sich vermindernd.

In solch schroffer Weise ist dies Verhalten neuerdings freilich kaum mehr ausgedrückt; und in dieser Weise wird es auch nicht von der Charcot'schen Schule formulirt.

Chareot selbst betont zunächst nur, dass das Zittern der Paralysis agitans bei Ruhe nicht aufhört, er sagt gelegentlich, dass es ebensowohl bei Ruhe der Glieder als bei intendirten Bewegungen zu beobachten sei; allerdings führt er kurz danach aus, dass der Tremor in bestimmten Fällen nur intermittirend auftrete, und hier finde er sich merkwürdiger Weise lediglich während der Ruhe und verschwinde beim Bewegen der Glieder.

In der neueren Zeit, seitdem eine grosse Zahl eingehender Krankenbeobachtungen vorliegen, behandeln die meisten Autoren die Frage nach der Art des Zitterns bei Paralysis agitans sehr vorsichtig. Es wird zwar das von Charcot beschriebene Verhalten als Regel hingestellt, 
doch werden eine Reihe von Ausnahmen zugelassen. So machen Gowers und Leube in ihren Lehrbiichern speciell darauf aufmerksam, dass ein Abweichen von jener Regel differentialdiagnostisch nicht verwerthbar sei; 0 ppenheim giebt wenigstens für die Fälle, in denen das Zittern überhaupt im Krankheitsbild zurücktritt, Intentionstremor zu.

Wohl die genauesten Krankengeschichten sind aus der Westphal'schen Klinik durch Heimann ${ }^{1}$ ) veröffentlicht. Da wo er die Symptome der Krankheit bespricht, sagt er nur, dass das Zittern nach allgemeiner Annahme auch in der Ruhe fortbestehe und durch Bewegungen sogar vermindert werden könne. Aus seinen eigenen Beobachtungen zieht er keine Schlüsse über diese Dinge; und gerade hier finden sich sehr gute Beschreibungen dieser Verhältnisse bei den einzelnen Kranken. Aus ihnen geht als sehr wesentlich hervor, dass die Art des Zitterns sehr wechselt. Bei einer Anzahl von Fällen wird angegeben, dass die Oscillationen in der Ruhe am stärksten sind, durch Ausfiuhrung willkürlicher Bewegungen deutlich verringert oder gar aufgehoben werden; bei einer gleich grossen Zahl nahm der Tremor bei activen Bewegungen sichtlich zu, und bei einer weiteren ebenso grossen Gruppe war das Verhalten beim einzelnen Kranken wechselnd.

Eine Durchsicht der Krankengesehichten aus der Klinik des Herrn Prof. Naunyn ergiebt ganz ähnliche Verhältnisse. Von 16 Fällen boten 7 den von Chareot beschriebenen Typus, 7 deutlichen Intentionstremor, 2 wechselnde Zustände.

Eine Anzahl casuistisch mitgetheilter Fälle, so bei A mid on (NewYork med. Ree. 1883), Demange (Revue de Med. 1882), eine dahin gehende Bemerkung bei Stephan (Arch. für Psych. XVIII. S. 757), enthält ebenfalls Angaben über das Vorkommen von Intentionstremor.

Ist dieser Intentionstremor bei Paralysis agitans nun wirklich identisch mit jenem bei multipler Sklerose, oder hat er vielleicht doch noch seine Eigenthẗmlichkeiten?

Dass bei demselben an Schüttellähmung leidenden Kranken das Zittern jeweilig ganz verschieden sein kann, wird allgemein angegeben. Viele dieser Patienten halten die Glieder ruhig, solange sie sich unbeobachtet füblen; jede psychische Aufregung ruft Zittern hervor oder steigert das vorhandene. Ebenso wird durch körperliche Anstrengung bei einer Reihe solcher Kranken das Zittern stärker. So beginnen bei Leuten, die gewöhnlich nur mit einem Arm zittern,

1) Ueber Paralysis agitans. Dissertation. Berlin 1888. 
die Oscillationen auch in anderen Gliedern, sowie sie mit einer Extremität irgend eine Kraftleistung auszuäben versuchen.

Diese beiden Momente, eine gewisse psychische Aufregung und eine körperliche Anstrengung, scheinen in der That wenigstens bei einem Theil der Kranken den stärkeren Tremor beim Bewegen der Glieder zu erklären. Sie sind offenbar von besonderem Einfluss bei solchen Patienten, bei welchen bald Intentionstremor, bald kein Intentionstremor beobachtet wird. In einem von Heimann's Fällen merkte der Kranke das Zittern zum ersten Male, als er bei einer Gerichtsverhandlung eine Unterschrift leisten sollte; einige Wochen später merkte er es wieder bei einer ähnlichen Gelegenheit.

Bei demselben Mann hörte das Zittern des rechten Armes auf, wenn er einen dicken Gegenstand umfasste; es hörte nicht auf, wenn er einen dünnen Stab nur mit zwei oder drei Fingern ergriff: bei complicirterer Bewegung, die eine feiner bemessene Muskelinnervation verlangte, bei der Patient seine Aufmerksamkeit genauer auf die Bewegung concentriren musste, dauerte das Zittern fort, das bei gröberer Action aufhörte. Beim selben Patienten lagen die Beine für gewöhnlich ruhig; sowie er sie steif ausgestreckt hielt, begannen sie zu zittern. In diesem Fall dürfte die grössere körperliche Anstrengung das veranlassende Moment sein. - Von einem anderen Kranken sagt Heimann, dass der Tremor merklich nachliess, wenn Patient mit der zitternden Extremität eine Bewegung ansführte; bei körperlichen Anstrengungen jedoch nahm der Tremor sofort zu, und zwar auch am linken Arm, wenn Patient mit dem rechten Arm einen kräftigen Druck ausübte.

Solche Fälle lassen daran denken, dass überall da, wo bei Paralysis agitans Intentionstremor beobachtet wird, das der Krankheit eigentlich zukommende Symptom nur verdeckt werde durch solche psychischen und somatischen Einflitsse, dass insbesondere der vom Arzt gegebene Befehl zur Ausführung einer Bewegung den Kranken regelmässig schon in gewisse Aufregung oder gar Verwirrung setze.

Indess hält diese einfache Erklärungsweise doch nur höchstens für einen Theil der Fälle Stand; denn man sieht typisches Intentionszittern auch bei geistig absolut stumpfen Patienten, welche durch eine noch dazu oft wiederholte ärztliche Untersuchung durchaus nicht in Aufregung kommen, und die auch keineswegs besondere Muskelanstrengungen dabei ausüben oder auch nur auszuüben glauben; man sieht es bei den Kranken auch dann zu Stande kommen, wenn sie sich ganz unbeobachtet glauben; und schliesslich ist die Steigerung des Zitterns durch psychische Erregung eine bei allen Tremorformen 
wiederkehrende Erscheinung; sie würde bei jeder anderen so gut wie bei der Paralysis agitans zur Erklärung des Intentionszitterns verwendet werden können. Gegen eine zu weite Ausdehnung eines solchen Erklärungsversuches spricht ohnehin das Verhalten bei der gewöhnlichen Form der Paralysis agitans mit Nichtintentionstremor.

Vielfach kehrt in den Krankengeschichten die Angabe wieder, dass während der Bewegung zwar der Tremor aufhöre oder sich doch verringere, dass er aber nach kurzer Pause wieder beginne. Nimmt diese Pause $a b$, und sind die ihr folgenden Oscillationen etwa besonders stark, dann könnte ein Intentionstremor vorgetänscht werden, obwohl im Grunde nur ein etwas verwischtes Bild der typischen Form vorlag, es liesse sich jedenfalls ein solches Verhalten mit der gangbaren Regel noch in Einklang bringen. Auch diese Deutung: mag für einzelne Fälle Geltung haben, für eine Reihe anderer gilt sie jedenfalls nicht. Das lässt sich am einfachsten an Curven zeigen. Die nachstehende graphische Darstellung stammt von einem Kranken,

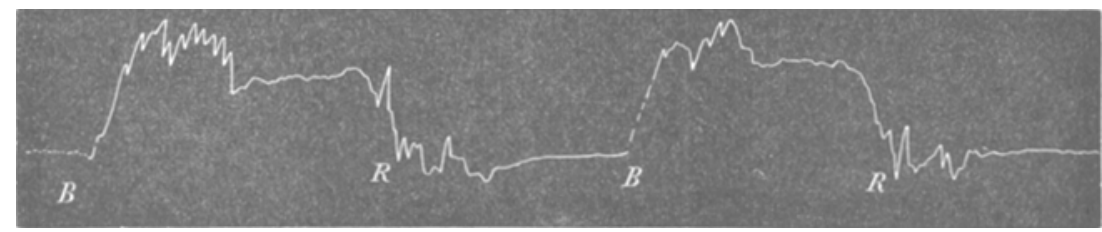

bei dem an der Diagnose kein Zweifel sein kann. Er hat die typische Haltung, die Muskelsteifigkeit, den Gang, die bekannte Schwierigkeit beim Aufstehen, beim Herumdrehen, die ständige Unruhe, Hitzegefühl an der Haut u. s. w. Wenn er ruhig sitzt, ist kein Tremor zu bemerken; beim Bewegen der Arme tritt regelmässig deutliches Zittern auf, links wesentlich stärker als rechts. Die Curve lässt deutlich erkennen, dass sogleich mit dem Ausstrecken des Armes (bei $B$ ) der Tremor beginnt, dass er aber unmittelbar nach dem Ausstrecken (bei $R$ ) des Gliedes aufhört; der Arm wird nun ganz still gestreckt gehalten. Mit dem Niederlassen des Armes tritt ein ähnlicher Tremor auf, der erst einige Secunden, nachdem die Extremität ihre Unterlage erreicht hat, zur Ruhe kommt. In derselben Weise bekommt Patient in den vorher ruhigen Händen deutlichen Tremor, wenn er einen Gegenstand mit der Fingerspitze berihren will. Der Tremor ist dabei so stark, dass Patient gewöhnlich ein paar Male am Ziel vorbeigreift. Hat er es erreicht, so kann er nun eine ganze Weile den Finger ruhig daran halten; doch tritt jetzt gewöholich auch Zittern auf, wenn man den Patienten auffordert, ja das Zittern zu vermeiden, oder auf den Gegen- 
stand mit dem Finger zu drücken. Beim Niederlegen des Armes kommt wiederum leichter Tremor. Fast regelmässig wird dieser Tremor weit stärker, wenn der Arm die Unterlage erreicht hat. Wenn Patient einige Male nach einander die Bewegung gemacht hat, tritt dies stärkere Zittern nach dem Wiedergelangen des Armes in die Ruhelage noch deutlicher hervor.

Ob dieser rasch wieder schwindende Tremor nach Bewegungen dem Zittern bei Körperruhe, welches die typischen Fälle haben, entspricht, oder ob er doch mit der ausgeführten Bewegung in mehr oder minder directem Zusammenhang steht, sei noch dahingestellt. Man könnte an die Anschauung von Ordenstein denken, dass das Zittern der Paralysis agitans ïberhaupt nicht Reiz-, sondern Lähmungserseheinung sei, hervorgerufen durch mangelhafte Innervation der bei der allgemeinen Steifigkeit ständig contrahirten Muskeln.

Die letzten, mir zur Beobachtung gekommenen Fälle, die speciell darauf hin untersucht wurden, boten dieses Symptom - rasch voruibergehende Steigerung der Oscillationen unmittelbar nachdem der Arm nach einer intendirten Bewegung wieder in Ruhelage gebracht worden - regelmässig ganz deutlich dar, und zwar sowohl die mit dem gewöhnlichen Typus als auch jene mit Intentionstremor. Vielleicht ist es noch am ehesten als charakteristisch für den Tremor bei Schüttellähmung zu brauchen, wenigstens gegenüber dem der multiplen Sklerose; bei gewissen Fällen einfachen senilen Tremors scheint ein ähnliches Verhalten vorzukommen.

Nach Allem glaube ich nicht, dass das Zittern der Paralysis agitans in so bestimmter Weise charakterisirt sei, wie das speciell von den ersten Bearbeitern betont wurde. In den ganz ausgebildeten Fällen findet es sich häufig in der von $\mathrm{Charcot}$ beschriebenen Weise - Nachlass der in der Rube bestehenden Oscillationen bei Bewegungen; aber sowobl bei solchen „ausgebildeten" Fällen, als auch bei anderen, die überhaupt nur geringen Tremor zeigen, kommt hänfig genug eine Modification des Zitterns vor, die als richtiger Intentionstremor bezeichnet werden muss.

Freilich wird man in den meisten Fällen die Unterscheidung von der multiplen Sklerose obne grosse Mühe machen können. Wenn die Bewegungen bei dieser Form der Schüttellähmung auch keineswegs immer so regelmässig ausfallen, als es gemeinhin gelehrt wird, und an Intensität denen bei multipler Sklerose kaum nachstehen, ist doch die ganze Art der Bewegung in der Regel bei beiden Krankheiten eine verschiedene. Das auffallend Träge, Zögernde, zumal beim Beginn des Actes, ist schon ziemlich charakteristisch gegen- 
uiber dem hastigen, unsicheren Greifen bei der Sklerose en plâques. Viel sicherer aber, als die Art der Bewegungen wird immer die Beobachtung des Gesammtsymptomencomplexes die Differentialdiagnose stellen lassen; und ich glaube, dass in etwa zweifelhaften Fällen diese Differentialdiagnose leichter wird, wenn wir uns dartiber klar sind, dass dem „Cardinalsymptom" des Zitterns kein zu grosser Werth beizulegen ist. 